\title{
Tratamento de Aneurisma da Aorta Abdominal por PEVAR Complicado de Pseudo-Aneurisma Femoral
}

\section{Percutaneous Endovascular Abdominal Aortic Aneurysm Treatment, Complicated by Femoral Pseudo-Aneurysm}

Joel Sousa ${ }^{1,2}$, Daniel Brandão ${ }^{1,2}$, Paulo Barreto ${ }^{1}$, Joana Ferreira ${ }^{1}$, José Almeida-Lopes ${ }^{1,2}$, Armando Mansilha ${ }^{1,2}$

\section{RESUMO}

O aneurisma da aorta abdominal constitui uma patologia comum e silenciosa, com desfechos potencialmente trágicos em situação de rutura. Se no passado a sua correção requeria abordagens cirúrgicas abdominais complexas, com grande morbi-mortalidade associada, o advento da correção endovascular do aneurisma da aorta abdominal (EVAR) e a sua generalização como procedimento gold-standard para o tratamento desta patologia vieram revolucionar o estado da arte, com possibilidade de tratamento de pacientes progressivamente mais velhos ou de risco cirúrgico previamente considerado proibitivo. Este é um caso clínico de um doente de 86 anos de idade, com múltiplos fatores de risco cardiovascular, submetido a EVAR sob anestesia local com acesso por via percutânea para correção de um aneurisma da aorta abdominal, o qual se complicou de pseudo-aneurisma femoral esquerdo, corrigido de forma minimamente invasiva e com sucesso por injeção percutânea ecoguiada de trombina.

PALAVRAS-CHAVE: Aorta Abdominal; Artéria Femoral; Embolização Terapêutica; Procedimentos Endovasculares; Trombina

\section{ABSTRACT}

Abdominal aortic aneurysm is a common and silent disease, with potentially tragic outcomes in case of rupture. Although in the past the treatment of this pathology always required complex surgical procedures, with great perioperative morbidity and mortality, the development of the endovascular aneurysm repair (EVAR) techniques, and their generalization as gold-standard procedures, completely revolutionized the current state of the art. Nowadays, and due to these techniques, it is possible to treat older patients as well as patients with higher surgical risk, previously deemed unfit for surgery. This is a case report of an 86 years old male, with multiple cardiovascular risk factors, diagnosed with an abdominal aortic aneurysm, and treated by EVAR with percutaneous access and under local anaesthesia. The procedure complicated with the development of a left femoral pseudo-aneurysm, which was successfully corrected in a minimally invasive fashion through percutaneous echo-guided injection of thrombin.

KEYWORDS: Aortic Aneurysm, Abdominal; Embolization, Therapeutic; Endovascular Procedures; Femoral Artery; Thrombin 


\section{INTRODUÇÃO}

A primeira correção endovascular de aneurisma da aorta (EVAR) foi realizada em 1987 por Volodos et al, ${ }^{1}$ os quais procederam à implantação de um stent coberto na aorta torácica descente para correção de um aneurisma pós-traumático. Se no início esta técnica constituía uma opção terapêutica de exclusão, as menores taxas de internamento e morbi-mortalidade pós-operatórias constatadas nos múltiplos estudos que desde então decorreram sobre a técnica, levaram a que praticamente se tenha tornado no gold-standard terapêutico, à luz daquilo que são as melhores práticas atuais. ${ }^{2,3}$

Classicamente, a realização deste tipo de implantes requeria a total exposição cirúrgica da artéria femoral (por norma, bilateralmente), tendo em vista garantir uma correta manipulação do material a implantar durante o procedimento e hemostase adequada no final do mesmo. ${ }^{4}$ Embora acarretem baixo risco cirúrgico para o doente, este tipo de exposições não estão desprovidas de riscos, com complicações locais descritas em 14-22\% dos casos, as quais, inevitavelmente, constituem causa de maior morbilidade para o doente e taxa de reinternamento mais elevada neste grupo populacional tipicamente tão frágil. ${ }^{5,6}$

Tendo em vista minorar estas complicações de acesso, Haas et al descreveram em 1999 e pela primeira vez o EVAR com acesso por via percutânea (pEVAR).? A sua técnica baseia-se na utilização de dispositivos de encerramento percutâneo dependentes de sutura, os quais, ao permitirem o encerramento remoto do orifício de arteriotomia, obviam a necessidade de exposição femoral cirúrgica e as complicações que à mesma acrescem, conforme foi mais tarde comprovado num conhecido ensaio prospetivo, randomizado e controlado. ${ }^{8}$

Apesar das múltiplas vantagens do acesso percutâneo no tratamento endovascular do aneurisma da aorta abdominal, também aqui existe risco de intercorrências, com falência técnica reportada em $6 \%$ dos casos de encerramento com dispositivo de encerramento percutâneo proglide (Abbott Vascular, Redwood City, CA) e consequentes complicações potenciais, sejam elas hemorrágicas (hematoma, pseudo-aneurisma), ou trombóticas (trombose, dissecção arterial, embolização). ${ }^{8}$ Embora a exposição femoral cirúrgica seja frequentemente necessária na correção destas mesmas complicações, existem agora opções terapêuticas não invasivas, com elevadas taxas de eficácia descritas, mas naturalmente com aplicabilidade variável. Um exemplo de sucesso é a injeção percutânea de trombina no tratamento do pseudo-aneurisma femoral, a qual cursa com taxas de sucesso de $91 \%$, desde que a anatomia da lesão seja adequada, e a experiência do operador, assegurada.?

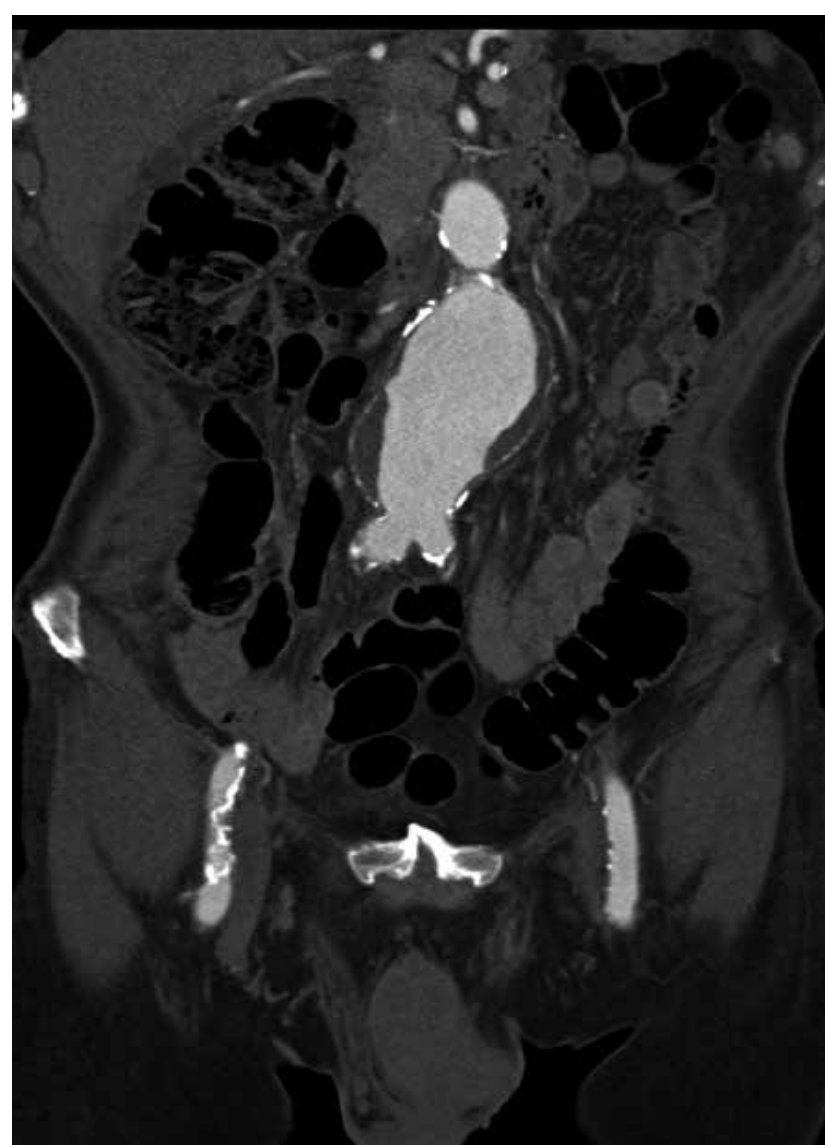

FIGURA 1. Corte coronal em angio-TC abdominopélvico, revelando aneurisma da aorta abdominal.

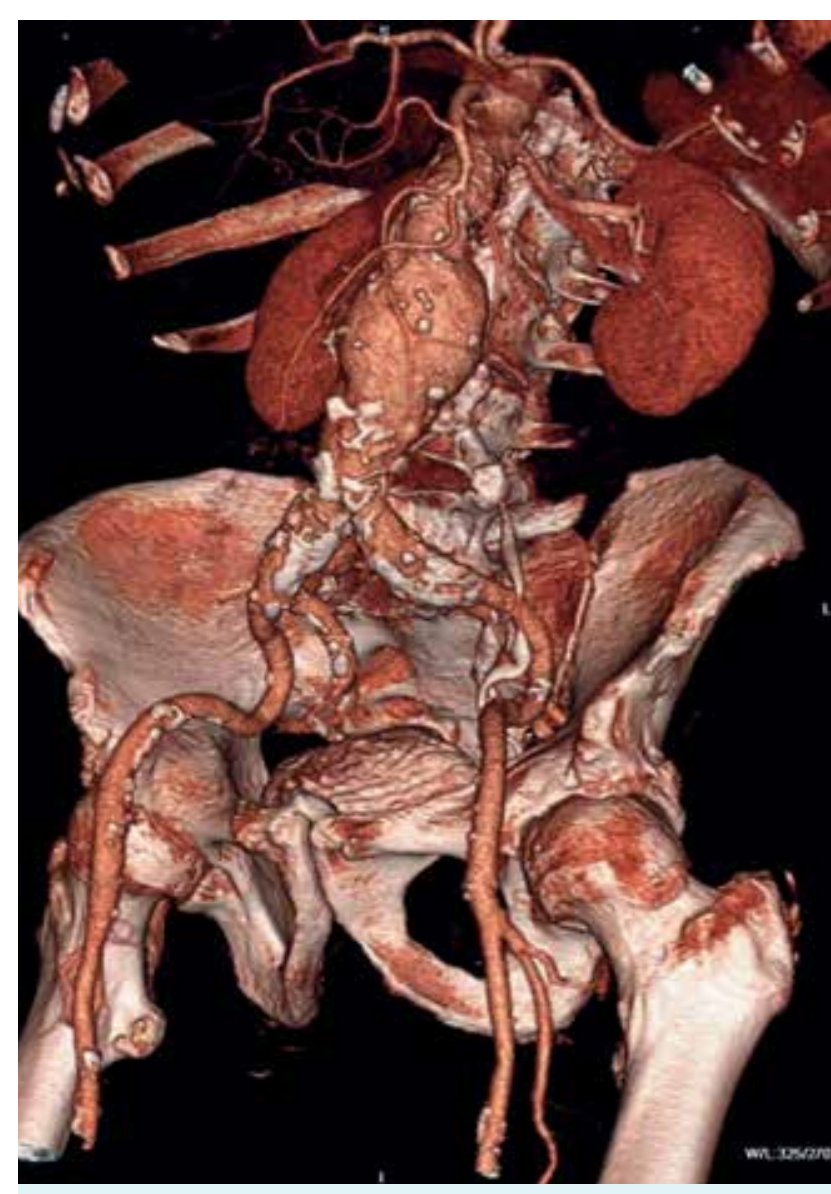

FIGURA 2. Reconstrução tridimensional em angio-TC do aneurisma da aorta abdominal. 


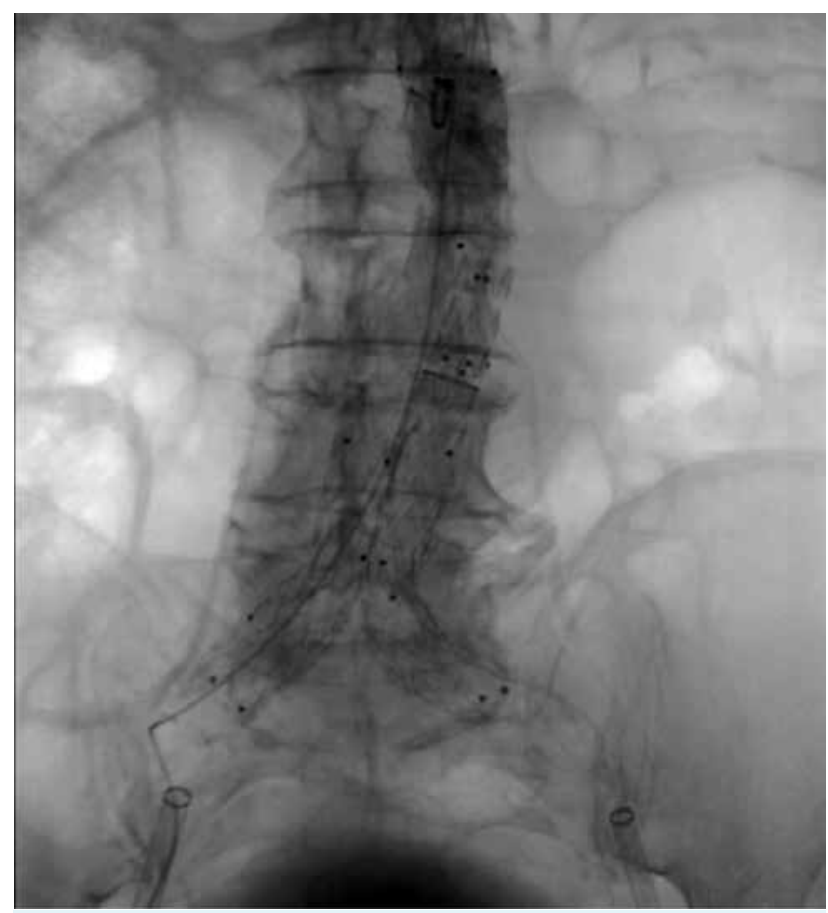

FIGURA 3. Angiografia intraoperatória revelando total patência da endoprótese, sem endoleaks visíveis no final do procedimento.

\section{CASO CLÍNICO}

Este é um caso clínico de um doente com 86 anos de idade, autónomo, com múltiplas comorbilidades cardiovasculares (hipertensão arterial; cardiopatia valvular por estenose aórtica moderada a severa; hipertrofia ventricular esquerda), regularmente acompanhado em consulta externa de Gastrenterologia por colite ulcerosa, devidamente medicada e controlada. No seguimento do estudo da sua doença inflamatória intestinal identificou-se, em angio-tomografia computorizada (TC) abdominopélvica de rotina, a presença de um aneurisma da aorta abdominal infrarrenal de $6,5 \mathrm{~cm}$ de maior diâmetro, assintomático (Figs. 1 e 2).

Atendendo à dimensão do aneurisma, o qual apresentava indubitável critério operatório, e tendo em conta o excelente estado geral do doente, decidiu-se, após discussão cuidada com o próprio e respetiva família, proceder-se ao tratamento do mesmo, por via endovascular, com recurso a EVAR com acesso por via percutânea (pEVAR) segundo a técnica de Preclose (Double Proglide - Abbott Vascular, Redwood City, CA). A intervenção foi realizada sob anestesia local com sedação consciente, tendo decorrido sem quaisquer complicações, com total exclusão do saco aneurismático, preservação das artérias renais e hipogástricas e sem quaisquer endoleaks objetiváveis na angiografia de controlo (Fig. 3).

No pós-operatório imediato o doente permaneceu clinicamente bem, sem evidência de nefropatia de contraste ou sinais de isquemia periférica. Ao $3^{\circ}$ dia pós-operató-

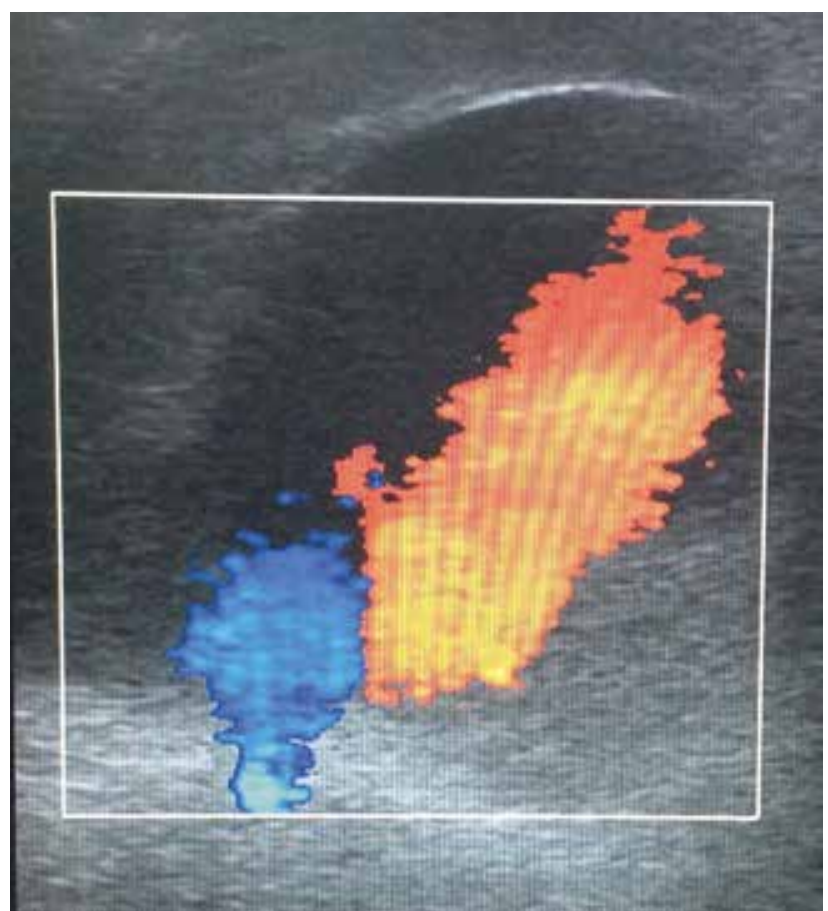

FIGURA 4. Volumoso pseudo-aneurisma femoral, evidente ao eco-Doppler (corte transversal).

rio, perante a constatação de expansibilidade anormal na zona de punção femoral esquerda, realizou-se estudo ultrassonográfico de controlo, o qual demonstrou a presença de um volumoso pseudo-aneurisma da artéria femoral comum com aproximadamente $3,5 \mathrm{~cm}$ de maior diâmetro e colo de 12 mm de comprimento por 2-3 mm de diâmetro (Fig. 4).

Atendendo às múltiplas comorbilidades do doente, e tendo em conta o risco de rutura do pseudo-aneurisma, decidiu-se pela tentativa de correção do mesmo de forma não invasiva, com recurso a injeção percutânea ecoguiada de trombina. O procedimento decorreu sem intercorrências e com aparente total exclusão da lesão no final do mesmo, tendo o doente permanecido com penso compressivo com vista à reavaliação às $24 \mathrm{~h}$. Aquando da reavaliação constatou-se contudo repermeabilização parcial do saco pseudo-aneurismático, pelo que se optou pela manutenção de medidas de tratamento conservador com penso compressivo e reavaliação diária. Ao $7^{\circ}$ dia pós-operatório, e perante a falência persistente do tratamento conservador, decidiu-se por nova injeção percutânea ecoguiada de trombina, desta feita com sucesso, com total exclusão do saco pseudo-aneurismático comprovada imagiologicamente (Fig. 5).

Ao $10^{\circ}$ dia pós-operatório, após estabilização das Comorbilidades médicas e total correção da complicação cirúrgica descrita, o doente teve alta para o domicílio orientado para a consulta da especialidade, onde tem vindo a ser acompanhado desde então. 


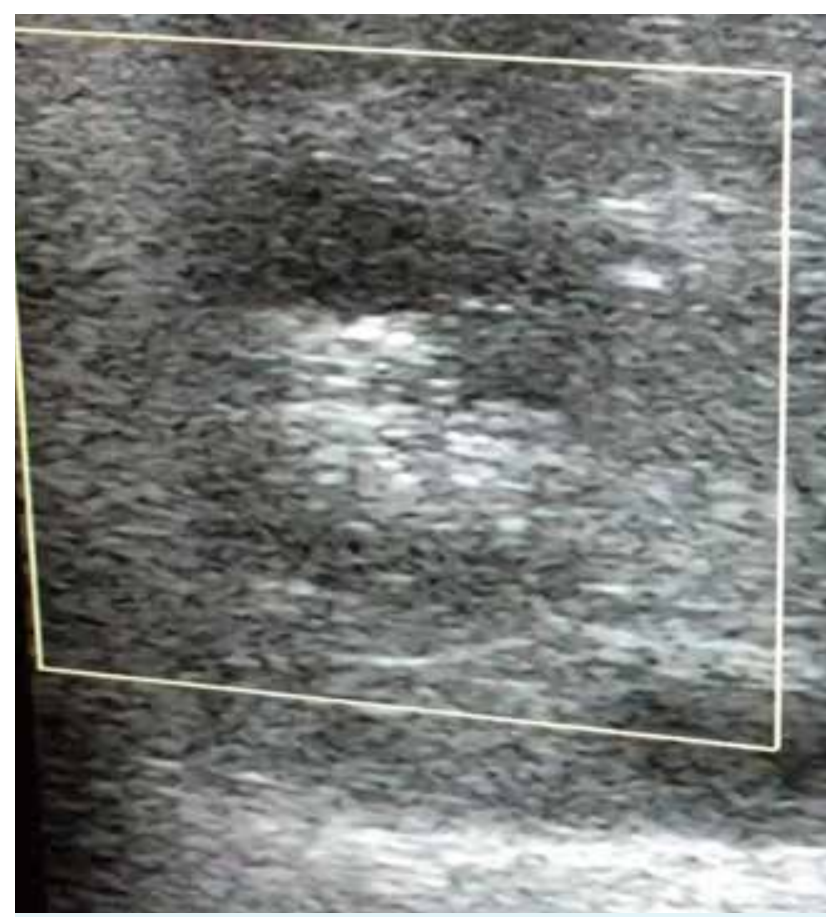

FIGURA 5. Pseudo-aneurisma totalmente excluído no final da intervenção.

\section{DISCUSSÃO}

aneurisma da aorta abdominal constitui uma patologia silenciosa e temível, com taxas de mortalidade que rondam os 90\% quando em situação de rutura. ${ }^{10}$ Considera-se que apresenta critério operatório quando o seu diâmetro transversal é superior a $5,5 \mathrm{~cm}$ ou se documenta um crescimento superior a $0,5 \mathrm{~cm}$ no período de seis meses, independentemente de qual o seu diâmetro. ${ }^{11}$ A sua identificação precoce e correção atempada constituem assim uma necessidade, numa tentativa de prevenir um desfecho potencialmente trágico.

A introdução do EVAR como opção terapêutica para esta patologia veio reduzir drasticamente a morbilidade e mortalidade observadas na cirurgia convencional,12 permitindo desta forma o tratamento eletivo de pacientes com idade progressivamente mais avançada ou risco cirúrgico mais elevado. O recurso à via de acesso percutâneo constituiu um avanço notável desta técnica, obviando assim a necessidade de exposição femoral cirúrgica e consequentemente todas as complicações que frequentemente daí advinham.

Embora as suas vantagens sejam indiscutíveis, mesmo esta via de acesso minimamente invasiva não é desprovida de complicações, de entre as quais se destaca o pseudo-aneurisma femoral como uma das mais comuns e mais graves. De facto, a sua formação cursa com um risco significativo de rutura e hemorragia, pelo que normalmente requer tratamento urgente. ${ }^{13}$

Dependendo das suas características anatómicas, o tra- tamento do pseudo-aneurisma femoral poderá passar da simples compressão extrínseca até à própria correção cirúrgica com exposição femoral, a qual é geralmente reservada para as lesões mais volumosas ou com colo mais largo. ${ }^{14}$ Se no passado o tratamento do pseudo-aneurisma femoral não passível de tratamento conservador requeria necessariamente a sua correção cirúrgica, a validação da injeção percutânea de trombina como opção terapêutica desta complicação veio obviar esta necessidade, com benefícios previsíveis, atendendo ao elevado risco cirúrgico e faixa etária da grande maioria destes doentes.

Os repetidos avanços nesta área têm assim permitido o tratamento com sucesso do aneurisma da aorta abdominal e suas complicações de acesso em doentes com múltiplas comorbilidades, garantindo taxas de morbilidade e mortalidade mínimas, aliadas a um maior conforto ao longo de todo o processo. O presente caso clínico constitui assim um excelente exemplo disso mesmo.

CONFLITOS DE INTERESSE: Os autores declaram a inexistência de conflitos de interesse na realização do trabalho.

FONTES DE FINANCIAMENTO: Não existiram fontes externas de financiamento para a realização deste artigo.

CONFIDENCIALIDADE DOS DADOS: Os autores declaram ter seguido os protocolos do seu centro de trabalho acerca da publicação dos dados de doentes.

PROTEÇÃO DE PESSOAS E ANIMAIS: Os autores declaram que os procedimentos seguidos na elaboração do presente trabalho estão em conformidade com as normas das comissões de investigação clínica e de ética, bem como da declaração de Helsínquia e da Associação Médica Mundial.

\section{REFERÊNCIAS}

1. Volodos NL KI, Troyan VI, Kalashnikova YuV, Shekhanin VE, Ternyuk NE, et al. Clinical experience of the use of self-fixing synthetic prostheses for remote endoprosthetics of the thoracic and the abdominal aorta and iliac arteries through the femoral artery and as intraoperative endprosthesis for aorta reconstruction. Vasa Suppl. 1991;33:93-5.

2. Dua A, Kuy S, Lee CJ, Upchurch GR, Jr., Desai SS. Epidemiology of aortic aneurysm repair in the United States from 2000 to 2010. J Vasc Surg. 2014;59:1512-7.

3. Moscato VP, O'Brien-Irr MS, Dryjski ML, Dosluoglu HH, Cherr GS, Harris LM. Potential clinical feasibility and financial impact of same-day discharge in patients undergoing endovascular aortic repair for elective infrarenal aortic aneurysm. J Vasc Surg. 2015;62:855-61.

4. Morasch MD. Percutaneous techniques for aneurysm repair. J Vasc Surg. 2006:43 Suppl A:69A-72A.

5. Lonn L, Larzon T, Van Den Berg JC. From puncture to closure of the common femoral artery in endovascular aortic repair. J Cardiovasc Surg. 2010;51:791-8. 
6. Maleux G, Koolen M, Heye S. Complications after endovascular aneurysm repair. Semin Intervent Radiol. 2009;26:3-9.

7. Haas PC, Krajcer Z, Diethrich EB. Closure of large percutaneous access sites using the Prostar XL Percutaneous Vascular Surgery device. J Endovasc Surg. 1999;6:168-70.

8. Nelson PR, Kracjer Z, Kansal N, Rao V, Bianchi C, Hashemi $\mathrm{H}$, et al. A multicenter, randomized, controlled trial of totally percutaneous access versus open femoral exposure for endovascular aortic aneurysm repair (the PEVAR trial). J Vasc Surg. 2014;59:1181-93.

9. Kuma S, Morisaki K, Kodama A, Guntani A, Fukunaga R, Soga Y, et al. Ultrasound-guided percutaneous thrombin injection for post-catheterization pseudoaneurysm. Circ J. 2015;79:127781.

10. Assar AN, Zarins CK. Ruptured abdominal aortic aneurysm: a surgical emergency with many clinical presentations. Postgrad Med J. 2009;85:268-73.
11. Aggarwal S, Qamar A, Sharma V, Sharma A. Abdominal aortic aneurysm: A comprehensive review. Exp Clin Cardiol. 2011;16:11-5

12. Propper BW, Abularrage CJ. Long-term safety and efficacy of endovascular abdominal aortic aneurysm repair. Vasc Health Risk Manag. 2013;9:135-41.

13. Pagliariccio G, Catalini R, Giantomassi L, Angelini A. Management of pseudoaneurysm of the leg: is Color Doppler US enough? J Ultrasound. 2010;13:61-5.

14. Huseyin S, Yuksel V. Sivri N, Gur O, Gurkan S, Canbaz S, et al. Surgical management of iatrogenic femoral artery pseudoaneurysms: A 10-year experience. Hippokratia. 2013;17:332-6. 